\title{
RELATIVE INTERIORS OF CONVEX HULLS
}

\author{
WILLIAM E. BONNICE ${ }^{1}$ AND JOHN R. REAY ${ }^{2}$
}

The purpose of this paper is to review several recent generalizations of a classic theorem of $\mathrm{E}$. Steinitz, to show how they are related, and to prove an extension of the Bonnice-Klee theorem which both generalizes and unifies these results. The theorem of Steinitz [6] is

Theorem A. If $A \subset R^{n}$ and $w \in$ int conv $A$, then $w \in$ int conv $B$ for some subset $B \subset A$ with card $B \leqq 2 n$.

Generalizations of this theorem have either tried to characterize when an upper bound of $2 n, 2 n-1$, etc. for card $B$ is necessarily assumed (see [4], [5]), have added further conditions on the set $A$ in order to obtain better bounds on card $B$ (see [1]-[4]), or have asked for the bounds on card $B$ if we demand only $w \in$ int $_{d} \operatorname{conv} B$ where $0 \leqq d \leqq n$. (Definition: $w \in$ int $_{d} X$ if there is a $d$-simplex contained in $X$ with $w$ in its relative interior.) The following two results are of the latter two types and are due respectively to Bonnice-Klee [1] and Ives [3].

THEOREM B. If $A \subset R^{n}$ and $w \in$ int $_{d} \operatorname{conv} A$ then $w \in$ int $_{d} \operatorname{conv} B$ for some subset $B \subset A$ with card $B \leqq \max (n+1,2 d)$.

Theorem C. If $A \subset R^{n}$ and $w \in \operatorname{int} \operatorname{conv} A$, let $B$ be a subset of $A$ of least cardinality such that $w \in \operatorname{int} \operatorname{conv} B$ and let $k$ be the dimension of the highest dimensional simplex with vertices in $A$ and having $w$ in its relative interior. Then

$$
n+1+\{n / k\} \leqq \operatorname{card} B \leqq 2 n-k+1 .
$$

( $\{x\}$ will always denote the largest integer strictly less than $x$.)

The extension we shall prove is

Theorem D. If $A \subset R^{n}$ and $w \in$ int $_{d} \operatorname{conv} A,(0 \leqq d \leqq n)$, let $B$ be a subset of $A$ of least cardinality such that $w \in$ int $_{d}$ conv $B$ and let $k$ be (as in Theorem $\mathrm{C}$ ) the dimension of the highest dimensional simplex with

Presented to the Society, April 22, 1967 under the title On a theorem of R. T. Ives; received by the editors May $23,1967$.

${ }^{1}$ Research supported in part by the National Research Council of Canada.

2 Research supported by Western Washington State College Bureau for Faculty Research. 
vertices in $A$ and having $w$ in its relative interior. If $d \leqq k$, then

$$
d+1 \leqq \operatorname{card} B \leqq k+1
$$

and if $k<d$, then

$$
d+1+\{d / k\} \leqq \operatorname{card} B \leqq \max (2 d-k+1, \min (2 k+2, n+2)) .
$$

Furthermore these bounds are as sharp as possible under the given conditions.

Since the proof of Theorem D depends upon Theorem C, we will give an independent proof of that result. The upper bound on card $B$ in Theorem $\mathrm{C}$ was established independently in [2]. The proof of the lower bound on card $B$ in Theorem $C$ as reported by Ives [3] was based on an induction on $k$. The proof below of the lower bound is straightforward and is an easy consequence of the following useful result in the theory of positive bases $[4$, Theorem 2.6, p. 11]. Also see [5].

Lemma E. Let $B$ be a positive basis for $R^{n}$. Then $B$ admits a partitioning $B=V_{1} \cup \cdots \cup V_{r}$ into pairwise disjoint subsets $(1 \leqq r \leqq n)$, such that

(1) card $V_{1} \geqq$ card $V_{2} \geqq \cdots \cdots$ card $V_{r} \geqq 2$.

(2) For each $j, 1 \leqq j \leqq r$ the set pos $\left(V_{1} \cup \cdots \cup V_{j}\right)$ is a linear subspace of $R^{n}$ of dimension $\sum_{i=1}^{j}\left(\operatorname{card} V_{i}-1\right)$.

Proof of Theorem C. Let $p$ denote $\operatorname{card} B$. We show only that $n+1+\{n / k\} \leqq p$. Without loss of generality assume $w$ is the origin. Then $p$ has the equivalent definition

$$
p=\min \left\{\operatorname{card} B \mid B \subset A, B \text { is a positive basis for } R^{n}\right\} .
$$

Since this minimum is assumed for some set $B$, let us now assume that $B$ is a positive basis for $R^{n}, B \subset A$, card $B=p$, and there is no smaller subset of $A$ which forms a positive basis for $R^{n}$. Let $B$ $=V_{1} \cup \ldots \cup V_{r}$ be the partitioning of Lemma $\mathrm{E}$.

Since pos $V_{1}$ is a linear space of dimension (card $V_{1}-1$ ), it follows that card $V_{1} \leqq k+1$ by the definition of $k$. Since $n=\sum_{i=1}^{r}\left(\right.$ card $\left.V_{i}-1\right)$ $=\operatorname{card} B-r$ is fixed, we see that $p=\operatorname{card} B=n+r$ is a minimum when $r$ is as small as possible. It is clear that this occurs when each pos $V_{i}$ is a linear subspace of maximal dimension and $R^{n}$ is their direct linear sum; that is, card $V_{1}=$ card $V_{2}=\cdots$ = card $V_{r-1}$ $=k+1$, and card $V_{r}-1$ is either $k+1$ or the remainder after dividing $n$ by $k$. Then $r=\{n / k\}+1$. Thus $p=n+r=n+\{n / k\}+1$ as was to be shown. 
Proof of Theorem D.

Case 1: $(d \leqq k)$. If $w \in$ int $_{d}$ conv $B$, then clearly card $B \geqq d+1$. If $A$ is a set of exactly $k+d+2$ points, if $k+d \leqq n$, and if $A=A_{1} \cup A_{2}$ where card $A_{1}=k+1$, card $A_{2}=d+1$, and the $k$-dimensional flat aff $A_{1}$ meets the $d$-dimensional flat aff $A_{2}$ only at the point $\{w\}$, then $d+1=\operatorname{card} B$.

Clearly card $B \leqq k+1$, and if $A$ is the set of vertices of a $k$-simplex which has $w$ in its relative interior then card $B=k+1$. Thus these bounds are the best possible.

Case 2: $(k<d)$. Without loss of generality assume $w=0$, the origin, let $p=\operatorname{card} B$, and let $B=\left\{b_{i}\right\}_{i=1}^{p}$. Because $0 \in$ int $_{d}$ conv $B$ there are scalars $\mu_{i}>0\left(\mu_{i} \neq 0\right.$ by the minimality of $\left.B\right)$ with $\sum_{i=1}^{p} \mu_{i}=1$ such that $0=\sum_{i=1}^{p} \mu_{i} b_{i}$. Thus $-b_{j}=\sum_{i \neq j}\left(\mu_{i} / \mu_{j}\right) b_{i} \in \operatorname{pos} B$ for $j=1,2$, ..., p. Hence pos $B$ is closed under scalar multiplication and therefore is a linear subspace, say of dimension $m$. Then $0 \in \mathrm{int}_{m} \operatorname{conv} B$, and $m \geqq d$. To apply Theorem $C$ in pos $B$, we note that for no proper subset $C$ of $B$ is $0 \in$ int $_{m} \operatorname{conv} C$ ( $C$ int $_{d} \operatorname{conv} C$ ) and that if $j$ is the dimension of the highest dimensional simplex with vertices in $B$ and having 0 in its relative interior, then $j \leqq k$. Thus by Theorem $\mathrm{C}$, card $B \geqq m+1+\{m / j\} \geqq d+1+\{d / k\}$.

To establish the asserted upper bound for card $B$, let $S \subset A$ be the set of vertices of a $k$-simplex with 0 in its relative interior. Thus pos $S$ is a $k$-dimensional subspace of $R^{n}$. Extend $S$ to a positive basis $C \subset A$ of $R^{n}$.

Keeping in mind the maximality of $k$, an examination of the proof of Lemma E [5], [4, Theorem 2.6, p. 11] shows that $S$ can be used as $V_{1}$ in the factorization $C=V_{1} \cup \cdots \cup V_{r}$ as given by Lemma $E$. Thus $k+1=$ card $V_{1} \geqq \cdots \cdot$ card $V_{r} \geqq 2$ and for each $j, 1 \leqq j \leqq r$, the set $\operatorname{pos}\left(V_{1} \cup \ldots \cup V_{j}\right)$ is a linear subspace of $R^{n}$ of dimension $\sum_{i=1}^{j}\left(\right.$ card $\left.V_{i}-1\right)$. Let $j$ be the least integer such that $\sum_{i=1}^{j}\left(\operatorname{card} V_{i}-1\right) \geqq d$, and let $B^{\prime}=\bigcup_{i-1}^{j} V_{i}$. Then $0 \in$ int $_{d} \operatorname{conv} B^{\prime}$ and, by the minimality of $B$, card $B \leqq$ card $B^{\prime}$. Thus, to complete the proof, it suffices to show that

$$
\text { card } B^{\prime} \leqq \max (2 d-k+1, \min (2 k+2, n+2)) \text {. }
$$

If $j=2$, then $k+1=$ card $V_{1} \geqq$ card $V_{2}$ implies that card $B_{1}$ = card $V_{1}+$ card $V_{2} \leqq 2 k+2$. But also,

$$
n \geqq \operatorname{dim} \operatorname{pos} B^{\prime}=\left(\operatorname{card} V_{1}-1\right)+\left(\operatorname{card} V_{2}-1\right)=\operatorname{card} B^{\prime}-2
$$

so card $B^{\prime} \leqq n+2$. Therefore, if $j=2$, card $B^{\prime} \leqq \min (2 k+2, n+2)$.

Now suppose that $j \geqq 3$. From $d \geqq 1+\sum_{i=1}^{j-1}$ (card $V_{i}-1$ ), it follows that $2 d \geqq-2 j+4+2 \sum_{i=1}^{j-1}$ card $V_{i}$, and so 


$$
\begin{aligned}
2 d-k+1 & \geqq-2 j-k+5+\operatorname{card} V_{1}+\sum_{i=2}^{j-1} \operatorname{card} V_{i}+\sum_{i=1}^{j-1} \operatorname{card} V_{i} \\
& =-2 j+6+\operatorname{card} V_{2}+\sum_{i=3}^{j-1} \operatorname{card} V_{i}+\sum_{i=1}^{j-1} \operatorname{card} V_{i} \\
& \quad \quad\left(\text { since card } V_{1}=k+1\right) \\
\geqq-2 j+6+\operatorname{card} V_{2}+(j-3) 2+\sum_{i=1}^{j-1} \operatorname{card} V_{i} & \quad\left(\text { since always card } V_{i} \geqq 2\right) \\
\geqq \operatorname{card} V_{j}+\sum_{i=1}^{j-1} \operatorname{card} V_{i} & \\
& =\operatorname{card} B^{\prime} .
\end{aligned}
$$

Combining the above results for $j=2$ and $j \geqq 3$, we have that always card $B^{\prime} \leqq \max (2 d-k+1, \min (2 k+2, n+2))$.

ExAmples. Here we show the bounds of Theorem $D$ are the best possible under the given conditions for the case $k<d$. In each example let $R^{n}$ be the direct linear sum of $r$ linear subspaces $\operatorname{pos} A_{i}, i=1$, - . , r where $k+1=\operatorname{card} A_{1} \geqq \operatorname{card} A_{2} \geqq \cdots \geqq \operatorname{card} A_{r} \geqq 2$, and each $A_{i}$ is the vertex set of a simplex with 0 in its relative interior. In each example, let the given set $A$ of Theorem $\mathrm{D}$ be $A=A_{1} \cup \ldots \cup A_{\text {r }}$ and let $w=0$.

The lower bound. For any given value of $d$ (where $d>k$ ) let

$$
\begin{aligned}
\operatorname{card} A_{1} & =\operatorname{card} A_{2}=\cdots=\operatorname{card} A_{j-1}=k+1 \geqq \operatorname{card} A_{j} \geqq 2 \\
& =\operatorname{card} A_{j+1}=\cdots=\operatorname{card} A_{r}
\end{aligned}
$$

where $j$ is determined by $d=\sum_{i-1}^{j}$ (card $\left.A_{i}-1\right)$. Then set $B$ of Theorem $\mathrm{D}$ will have cardinality card $B=\sum_{i=1}^{j}$ card $A_{i}=d+j$ $=d+\{d / k\}+1$.

The upper bounds. (1) If $r=2$ and $d=k+1$ then card $B=k+1$ + card $V_{2}=n+2=\max (2 d-k+1, \min (2 k+2, n+2))$.

(2) If $r \geqq 2$, and $d=k+1=\operatorname{card} A_{i}$ for $i=1, \cdots, r$, then $n=r k$ and card $B=2 k+2=\max (2 d-k+1, \min (2 k+2, n+2))$.

(3) If $r=3,(k+1) / 2 \leqq \operatorname{card} A_{2}=\operatorname{card} A_{3}$ and $d=k+\operatorname{card} A_{2}$ then

$$
\begin{aligned}
\operatorname{card} B & =k+1+2 \operatorname{card} A_{2}=2 d-k+1 \\
& =\max (2 d-k+1, \min (2 k+2, n+2)) .
\end{aligned}
$$

(4) If $r \geqq 2$, and card $A_{2}=\cdots=$ card $A_{r}=2$, then 


$$
\begin{aligned}
\operatorname{card} B & =(k+1)+2(d-k)=2 d-k+1 \\
& =\max (2 d-k+1, \min (2 k+2, n+2)) .
\end{aligned}
$$

Finally, we note that Theorem 2.9 of [1] also gives upper bounds on card $B$ under the hypothesis of Theorem $D$ of this paper but example (2) above and Theorem D show that the conclusion as stated there is incorrect and should read "For $e+1 \leqq d \leqq n$ there is a $Y_{d} \subset X$ such that pos $Y_{d}$ is a linear subspace of dimension $\geqq d$ and card $Y_{d}$ $\leqq 2 d-e+1<2 d$ or card $Y_{d} \leqq n+2$."

\section{REFERENCES}

1. W. Bonnice and V. L. Klee, The generation of convex hulls, Math. Ann. 152 (1963), 1-29.

2. W. Bonnice and J. R. Reay, Interior points of convex hulls, Israel J. Math. 4 (1966), 243-248.

3. R. T. Ives, $A$ generalization of Steinitz' theorem, Abstract $640-47$, Notices Amer. Math. Soc. 13 (1966), 849.

4. J. R. Reay, Generalizations of a theorem of Caratheodory, Mem. Amer. Math. Soc. No. 54, 1965.

5. - , A new proof of the Bonnice-Klee theorem, Proc. Amer. Math. Soc. 16 (1965), 585-587.

6. E. Steinitz, Bedingt konvergente Reihen und konvexe Systeme. I, II, III, J. Reine Angew. Math. 143 (1913), 128-175; 144 (1914), 1-40; 146 (1916), 1-52.

University of New Hampshire, University of Western ONTARIo and Western Washington State College 